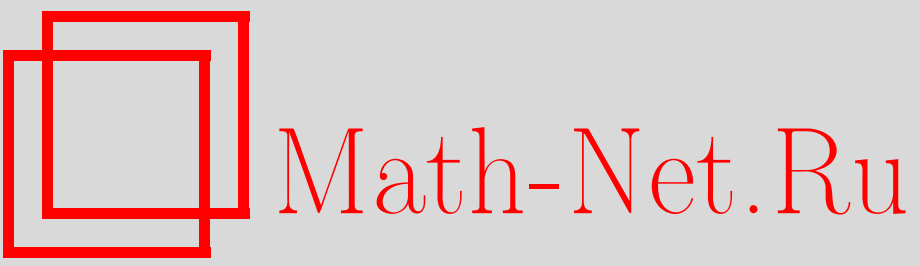

В. И. Сербин, Повышение эффективности процесса обучения с помощью марковской модели, Итоги науки и техн. Сер. Соврем. мат. и ее прил. Темат. обз., 2020, том 186, 116-122

DOI: https://doi.org/10.36535/0233-6723-2020-186-116-122

Использование Общероссийского математического портала Math-Net.Ru подразумевает, что вы прочитали и согласны с пользовательским соглашением

http://www.mathnet.ru/rus/agreement

Параметры загрузки:

IP: 54.80 .73 .141

26 апреля 2023 г., 14:17:24 


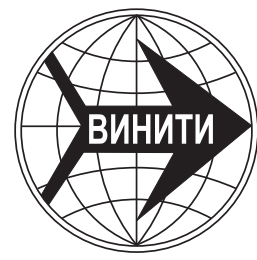

ИТОГИ НАУКИ И ТЕХНИКИ.

Современная математика и ее приложения.

Тематические обзоры.

Том 186 (2020). C. 116-122

DOI: 10.36535/0233-6723-2020-186-116-122

УДК 517.929

\title{
ПОВЫШЕНИЕ ЭФФЕКТИВНОСТИ ПРОЦЕССА ОБУЧЕНИЯ С ПОМОЩЬЮ МАРКОВСКОЙ МОДЕЛИ
}

\author{
(C) 2020 г. \\ В. И. СЕРБИН
}

\begin{abstract}
АннотАция. Рассматривается марковская модель обучения из трех состояний: состояние передачи знаний, состояние тренинга и состояние контроля знаний. Учет в рамках этой модели времени обучения позволяет получить дополнительную информацию о компетенциях обучаемых, оценить значения латентных параметров и на основе этого лучше управлять процессом обучения.
\end{abstract}

Ключевые слова: обучающая система, латентный параметр, тренинг, тестирование, математическая модель, марковская модель, дифференциальная энтропия, модель Раша.

\section{IMPROVING THE EFFICIENCY OF THE LEARNING PROCESS USING THE MARKOV MODEL}

\author{
(c) 2020 V. I. SERBIN
}

\begin{abstract}
We consider a Markovian model of learning consisting of three states: the state of knowledge transfer, the training state, and the state of knowledge control. Taking into account of training time within this model allows one to obtain additional information about competencies of trainees, to estimate the values of latent parameters, and to improve the learning process.
\end{abstract}

Keywords and phrases: training system, latent parameter, training, testing, mathematical model, Markov model, differential entropy, Rasch model.

AMS Subject Classification: 34K06, 34K10, 34K13

1. Введение. В работах Д. А. Новикова и других $[1,5,8]$ показано, что для повышения качества управления в различных социальных системах необходимо создание математической модели, позволяющей адекватно описывать, анализировать и управлять процессом обучения.

Управление учебным процессом, повышение эффективности обучения и, в частности, повышение уровня знаний, умений и навыков учеников, напрямую зависят от оперативного получения объективной и точной информации о результатах обучения на всех этапах передачи знаний, выработки умений и закреплении навыков. Решение этих задач зависит от адекватности и объективности оценок за обучение и латентных параметров обучения, таких как уровень подготовки ученика, трудность заданий тренинга и тестирования.

Наиболее распространенной формой контроля результатов обучения является тестирование, а его результаты оцениваются в дихотомических, а в лучшем случае, в порядковых шкалах. При этом время, затраченное на тестирование, или совсем не учитывается, или используется только как пороговое значение - для прекращения процесса тестирования.

Система тестированная, в которой оценка определяется по доле правильных ответов, также не является достаточно объективной, так как не учитывает ни уровня подготовки учеников, ни сложности заданий. Кроме того, результаты измерения в данной системе тестирования являются 


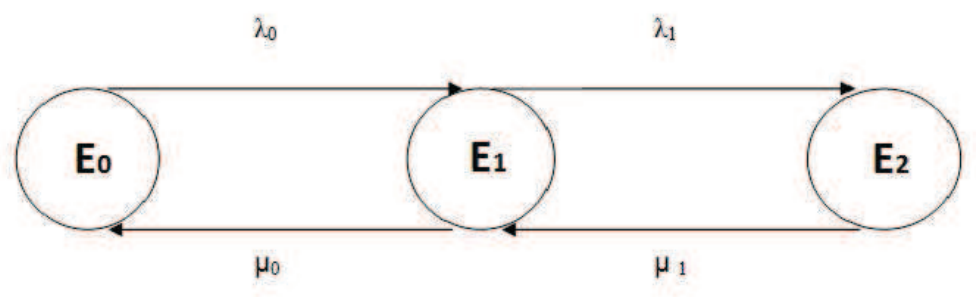

Рис. 1. Марковская модель обучающей системы из трех состояний

нелинейными, так как при определении уровня знаний «вес» одной решенной задачи тем выше, чем ближе число решенных задач к нулю или к общему количеству задач.

Системы тестирования, основанные на модели Раша [10], лишены этих недостатков, так как позволяют превратить измерения, сделанные в дихотомических и порядковых шкалах, в линейные измерения, которые затем можно проанализировать с помощью методов математической статистики, однако при этом оценка уровня подготовки учеников не зависит от трудности заданий, а оценка трудности заданий не зависит от уровня подготовки учеников.

Модель Раша, применяемая для обработки результатов тестирования и получения оценок, позволяет найти только отношение между независимыми латентными параметрами обучения: сложностью заданий и уровнем подготовки обучаемых - однако сами эти значения остаются неизвестными.

Учитывая все это, для повышения качества управления процессом обучения была предложена марковская модель оценки результатов обучения.

Объектом данного исследования является система измерения латентных параметров обучения на основе марковской модели.

Предмет исследования - оценка эффективности обучения на основе математической модели обучения, методы оценивания результатов обучения как средства управления процессом обучения и повышения качества обучения.

Цель исследования - повышение качества обучения за счет совершенствования управления процессом обучения.

Для достижения поставленной в исследовании цели были решены следующие задачи:

1. Выполнен системный анализ существующих моделей обучения, методов оценки результатов обучения, методов управления процессом обучения, исследованы структуры процесса обучения и определены факторы, влияющие на качество управления процессом обучения;

2. Разработана модель обучения, учитывающая время обучения и позволяющая определить латентные параметры обучения;

3. Разработан метод и сформирована процедура управления процессом обучения для повышения качества обучения.

В работе проведено исследование связи между моделью обучения, учитывающей время, и моделью Раша, разработан метод определения латентных параметров обучения и описан алгоритм управления процессом обучения на основе получаемых результатов тренинга и тестирований с учетом затраченного времени.

2. Основные результаты. Сформулируем основной результат работы.

Для решения задачи управления процессом обучения представим модель обучающей системы как систему с несколькими дискретными состояниями и непрерывным временем. Опишем эту модель как случайный марковский процесс, состоящий из следующих состояний: состояние передачи знаний, состояние решения тренировочных задач, или тренинга, и состояние контроля знаний, или тестирования.

На рисунке 1 изображена Марковская модель обучающей системы из трех состояний. 


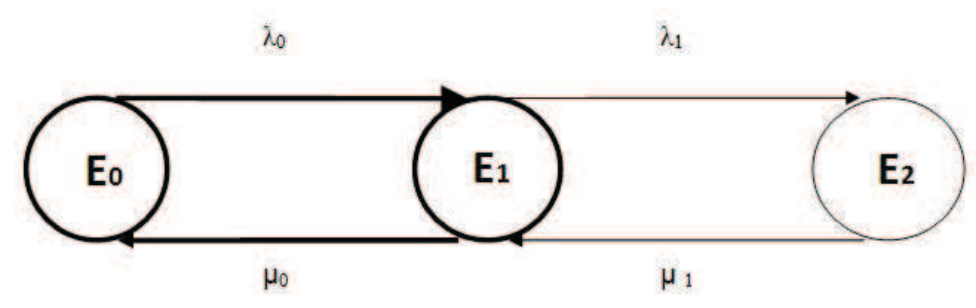

Рис. 2. Первый контур управления марковской моделью обучающей системы из трех состояний

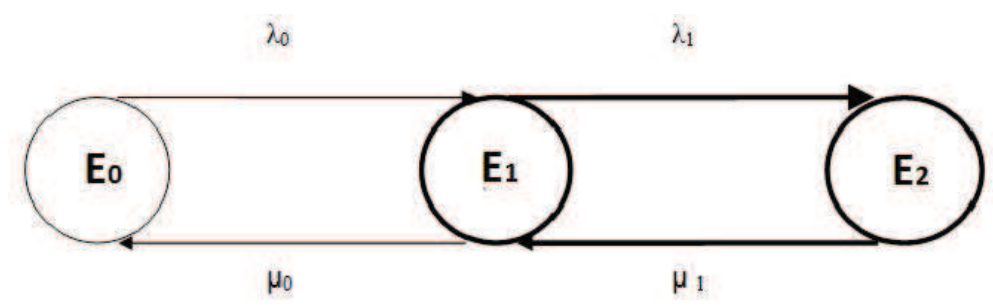

Рис. 3. Второй контур управления марковской моделью обучающей системы из трех состояний

Пусть вероятность того, что в момент времени $t$ система находится в состоянии $E_{i}$ равна $P_{i}(t)$, где $i=0,1,2$.

Тогда поведение системы во времени описывается системой уравнений Колмогорова

$$
\left\{\begin{array}{l}
\frac{d P_{0}(t)}{d t}=-\lambda_{0} P_{0}(t)+\mu_{0} P_{1}(t) \\
\frac{d P_{1}(t)}{d t}=\lambda_{0} P_{0}(t)-\left(\mu_{0}+\lambda_{1}\right) P_{1}(t)+\mu_{1} P_{2}(t) \\
\frac{d P_{2}(t)}{d t}=\lambda_{1} P_{1}(t)-\mu_{1} P_{2}(t)
\end{array}\right.
$$

В системе (1) время $t \geqslant 0$,

$E_{0}$ - состояние передачи знаний, или обучения,

$E_{1}$ - состояние тренинга,

$E_{2}$ - состояние тестирования,

$\lambda_{0}$ - интенсивность перехода из $E_{0}$ в $E_{1}$,

$\mu_{0}$ - интенсивность перехода из $E_{1}$ в $E_{0}$,

$\lambda_{1}$ - интенсивность перехода из $E_{1}$ в $E_{2}$,

$\mu_{1}$ - интенсивность перехода из $E_{2}$ в $E_{1}$.

Кроме того, должно выполняться нормировочное условие $P_{0}(t)+P_{1}(t)+P_{2}(t)=1$.

Данная система является марковским процессом гибели и размножения с тремя состояниями, непрерывным временем и постоянными интенсивностями.

Представим управление процессом обучения в виде двух контуров:

(1) контур обучение - тренинг $\left(E_{0} \Leftrightarrow E_{1}\right)$,

(2) контур тренинг - тестирование $\left(E_{1} \Leftrightarrow E_{2}\right)$.

На рис. 2 и 3 изображены первый и второй контуры марковской модели обучающей системы из трех состояний.

Рассмотрим работу системы на примере работы первого контура управления.

Данная модель описывает обучающую систему как случайный марковский процесс типа «гибель-размножение», состоящий из двух состояний: состояния обучения, или передачи знаний, и состояния тренинга [7]. 
Система уравнений Колмогорова, описывающих поведение системы во времени, будет иметь вид $[7,11]$

$$
\left\{\begin{array}{l}
\frac{d P_{0}(t)}{d t}=-\lambda_{0} P_{0}(t)+\mu_{0} P_{1}(t), \\
\frac{d P_{1}(t)}{d t}=\lambda_{0} P_{0}(t)-\mu_{0} P_{1}(t) .
\end{array}\right.
$$

Нормировочное условие $P_{0}(t)+P_{1}(t)=1$ для любого $t \geqslant 0$.

Данная модель описывает обучающую систему как случайный марковский процесс типа «гибель-размножение», состоящий из двух состояний: состояния активного обучения (передача знаний и тренинг) и состояния контроля знаний $[7,11]$.

Пусть случайная величина $T$ - время обучения. Тогда $P_{0}(t)=P(T<t)$ - вероятность успешного завершения процесса обучения не позже чем за время $t$; а $P_{1}(t)=1-P_{0}(t)=P(T \geqslant t)-$ вероятность успешного завершения процесса обучения не раньше чем за время $t$, или оценка за обучение. $\lambda_{0}$ - интенсивность состояния активного обучения, $\mu_{0}$ - интенсивность состояния решения задачи и контроля знаний.

Пусть работа системы начинается в состоянии $E_{1}$. Решив систему $(2)$ для $P_{0}(0)=0$ и $P_{1}(0)=1$, мы получим

$$
\left\{\begin{array}{l}
P_{0}(t)=P_{0}(\infty)\left(1-e^{-\left(\lambda_{0}+\mu_{0}\right) t}\right) \\
P_{1}(t)=1-P_{0}(t)
\end{array}\right.
$$

Величины

$$
\left\{\begin{array}{l}
P_{0}(\infty)=\lim _{t \rightarrow \infty} P_{0}(t)=\frac{\mu_{0}}{\lambda_{0}+\mu_{0}}, \\
P_{1}(\infty)=\lim _{t \rightarrow \infty} P_{1}(t)=\frac{\lambda_{0}}{\lambda_{0}+\mu_{0}}
\end{array}\right.
$$

- предельные значения параметров процесса.

Представим процесс обучения как процесс обработки информации или как последовательность элементарных операций преобразования данных (опд) [11].

Тогда интенсивность $\lambda_{0}$ - это количество опд, выполненных в единицу времени в состоянии обучения, или скорость обработки информации. Величину, обратную интенсивности $\sigma_{0}=1 / \lambda_{0}$ (среднее время выполнения одной опд), примем за уровень подготовки обучаемого.

Интенсивность $\mu_{0}$ - это количество опд, выполненных в единицу времени в состоянии тестирования, или скорость обработки информации. Величина, обратная интенсивности $\tau_{0}=1 / \mu_{0}-$ среднее время выполнения одной опд. Будем трактовать эту величину как трудность задачи (см. $[6,7,11])$.

Согласно аксиоме локальной независимости Лазарсфельда (см. [2]) будем считать величины $\lambda_{0}$ и $\mu_{0}$ независимыми.

Анализ формул (3) и (4) показывает, что функция распределения времени обучения равна $F_{\lambda_{0}}(t)=1-e^{-\lambda_{0} t}$, а плотность распределения $-f_{\lambda_{0}}(t)=\lambda_{0} e^{-\lambda_{0} t}$. Тогда дифференциальная энтропия, или среднее количество обработанной в процессе обучения информации, равна

$$
H(T)=\ln \frac{e}{\lambda_{0}}=\ln e \sigma_{0}=\beta,
$$

причем

$$
\sigma_{0}=e^{\beta-1} .
$$

Соответственно, функция распределения времени тренинга равна $F_{\mu_{0}}(t)=1-e^{-\mu_{0} t}$, плотность распределения $-f_{\mu_{0}}(t)=\mu_{0} e^{-\mu_{0} t}$, а дифференциальная энтропия, или среднее количество обработанной в процессе тренинга информации, равна

$$
H(T)=\ln \frac{e}{\mu_{0}}=\ln e \tau_{0}=\alpha
$$

и

$$
\tau_{0}=e^{\alpha-1}
$$


В итоге согласно формулам (5), (6), (7) и (8) предельное значение вероятности успешного решения заданий может быть представлено формулой

$$
P_{0}(\infty)=\frac{\mu_{0}}{\lambda_{0}+\mu_{0}}=\frac{1}{1+\lambda_{0} / \mu_{0}}=\frac{1}{1+\tau_{0} / \sigma_{0}}=\frac{1}{1+e^{\alpha-\beta}}
$$

Величины $\mu_{0}, \lambda_{0}, \tau_{0}, \sigma_{0}, \alpha$ и $\beta$ являются непрерывными латентными переменными (см. [9]); для их нахождения допустим, что в обучении принимает участие $n$ обучаемых, а каждый обучаемый решает $m$ задач. По результатам решения этих задач составим таблицу времени решения задач $T$ размером $n m$. Элемент таблицы $t_{i j}$ это время, потраченное $i$-м обучаемым на решение $j$-й задачи. Пусть $\lambda_{i}$ - интенсивность активного обучения $i$-го ученика, $\mu_{j}$ - интенсивность решения $j$-й задачи. В [6] методом наибольшего правдоподобия показано, что точечные оценки величин $\lambda_{i}$ и $\mu_{j}$ равны предельным значениям $\lambda_{i}^{(k)}$ и $\mu_{j}^{(k)}$ при $k \rightarrow \infty$. Значения $\lambda_{i}^{(k)}$ и $\mu_{j}^{(k)}$ находятся с помощью итерационных формул

$$
\left\{\begin{aligned}
\lambda_{i}^{(k+1)} & =\frac{\sum_{j=1}^{m} \frac{\lambda_{i}^{(k)}}{\lambda_{i}^{(k)}+\mu_{j}^{(k)}}}{\sum_{j=1}^{m} t_{i j}}, \quad i=1,2, \ldots, n \\
\mu_{j}^{(k+1)} & =\frac{\sum_{i=1}^{n} \frac{\mu_{i}^{(k)}}{\lambda_{i}^{(k)}+\mu_{j}^{(k)}}}{\sum_{i=1}^{n} t_{i j}}, \quad j=1,2, \ldots, m
\end{aligned}\right.
$$

при начальных значениях

$$
\left\{\begin{array}{l}
\lambda_{i}^{(0)}=\frac{m}{\sum_{j=1}^{m} t_{i j}}, \quad i=1,2, \ldots, n, \\
\mu_{j}^{(0)}=\frac{n}{\sum_{i=1}^{n} t_{i j}}, \quad j=1,2, \ldots, m .
\end{array}\right.
$$

3. Связь с моделью Раша. В однопараметрической модели обучения Раша (см. [10]) формула, определяющая вероятность того, что обучаемый с уровнем подготовки $s$ выполнит задание трудности $d$ - так называемая функция успеха - равна

или

$$
P(d, s)=\frac{1}{1+d / s}
$$

$$
P(d, s)=\frac{1}{1+e^{\delta-\theta}},
$$

где $d=e^{\delta}($ или $\delta=\ln d), s=e^{\theta}$ (или $\theta=\ln s$ ).

С точностью до обозначений формулы (12) и (13) совпадают с формулой $(9)$ для $P_{0}(\infty)$. Таким образом, модель обучения из двух состояний может рассматриваться как развитие модели Раша, а функцию успеха Раша целесообразно считать равной предельному значению вероятности успешного решения заданий $P_{0}(\infty)$.

4. Сравнительный анализ моделей. Пусть обучение проводится в три этапа, а обучающая система может находиться в одном из трех состояний: передачи знаний, выработки умений с помощью тренинга и проверки навыков с помощью тестирования.

Разница между тренингом и тестированием заключается в том, что в процессе тренинга задача должна быть обязательно решена. При этом решение задачи проводится под наблюдением и, если 
надо, с помощью учителя. Во время тестирования задача решается самостоятельно и должна быть решена как можно быстрее, но может быть и не решена.

В случае тренинга сложность задач должна быть не ниже уровня подготовки учеников, и в результате тренинга уровень подготовки должен повышаться. Сложность тестовых заданий может быть не выше уровня подготовки учеников, так как в эти задания входят задачи, которые обучаемый уже должен уметь решать, а при проверке навыков важен фактор скорости решения задач. Выбор соотношений между уровнем сложности заданий и уровнем подготовки обучаемых на этапах тренинга и тестирования является самостоятельной задачей. Эти соотношения можно подобрать, например, одним из итерационных методов.

Пусть в процессе обучения принимает участие $n$ обучаемых. После проведения каждого этапа обучения необходимо проверить результат с помощью проверочных заданий. Пусть эти задания состоят из $m$ вопросов или задач. По результатам испытаний составим две таблицы, состоящие из $n$ строк и $m$ столбцов: таблицу $A$ и таблицу $T$. Таблица $A$ содержит результаты по дихотомическому принципу $a_{i j}=1$ если $i$-й ученик правильно выполнил $j$-е задание, иначе $a_{i j}=0$. Таблица $T$ содержит время решения задачи. $t_{i j}$ - время, потраченное $i$-м учеником на выполнение $j$-го задания. Обработка результатов решения задач проводится по формулам (10), (11).

Рассмотрим особенности обработки полученных результатов в модели Раша и в модели обучающей системы из двух состояний.

Модель Раша, используемая для определения латентных параметров является одним из разделов теории латентного анализа Лазарсфельда (см. [2]), целью которого была обработка результатов социологических опросов. По результатам таких опросов составлялись таблицы, построенные по дихотомическому принципу. Поэтому в модели Раша используется только таблица $A$, которая называется матрицей ответов. Перед обработкой из нее удаляются строки и столбцы, содержащие все нули и все единицы. После этого производится обработки таблицы, при этом используется логистическая однопараметрическая модель Раша в модификации Ю. М. Неймана (см. [4]).

Таким образом, модель Раша не подходит для оценки результатов тренинга, в процессе которого ученик должен решить все задачи, а таблица результатов $A$ состоит из одних единиц.

В случае тестирования, при котором оцениваются навыки, время решения задач становится решающим фактором получения оценки, так как навыки предполагают не только и не столько умение правильно решать задачи (что отражается в таблице $A$ ), но и решать их за приемлемое время (что отражается в таблице $T$ ). Следовательно, можно ожидать, что таблица $A$ результатов тестирования будет содержать много строк и столбцов, состоящих из одних единиц. Это делает модель Раша менее пригодной для оценки результатов тестирования, чем модель обучающей системы из двух состояний.

5. Управление процессом обучения. На основании полученных результатов предлагается следующая процедура работы обучающей системы.

Перед началом обучения выполняется отбор заданий и входное тестирование учеников с целью определения уровней их подготовки. После этого проводится передача знаний, тренинг и тестирование для определения уровня знаний на основе использования тестовых заданий. При проведении тренинга примем за аксиому закон Йеркса-Додсона, который утверждает, что «по мере увеличения интенсивности мотивации качество деятельности изменяется по колоколообразной кривой: сначала повышается, а затем постепенно снижается» (см. [9]). Поэтому выполняется решение последовательности задач, упорядоченных по возрастанию трудности. Вначале решаются задачи, трудность которых совпадает с текущим уровнем подготовки обучаемых, затем оценивается уровень подготовки обучаемых. Если он повысился, то выбирается следующая последовательность задач с более высоким значением трудности и тренинг продолжается. Процесс прекращается, когда на очередном этапе решение задач не приводит к повышению уровня подготовки обучаемых. Предполагается, что достигнут «максимальный» результат тренинга.

После тренинга выполняется тестирование с помощью задач, трудность которых равна текущему уровню подготовки обучаемых и определяется итоговый уровень подготовки обучаемых. 


\section{СПИСОК ЛИТЕРАТУРЫ}

1. Данилова C. Д. Адаптивная нечеткая модель оценивания результатов автоматизированного тестирования с разделением заданий по уровням усвоения/ Дисс. на соиск. уч. степ. канд. техн. наук. Улан-Удэ: ВСГТУ, 2005.

2. Лазарсфельд П. Ф. Латентно-структурный анализ и теория тестов// в кн.: Математические методы в социальных науках. - М.: Прогресс, 1973. - С. 42-53.

3. Малкин И. Г. Теория устойчивости движения. - М.: Наука, 1966.

4. Нейман Ю. $M$, Хлебников B. А. Введение в теорию моделирования и параметризацию педагогических тестов. - М.: Прометей, 2000.

5. Новиков Д. А. Теория управления организационными системами. - М.: МПСИ, 2005.

6. Сербин В. И. Метод расчета параметров в автоматизированной обучающей системе// Прикасп. ж. Управл. высокие техн. - 2012. - 2 (18). - С. 66-70.

7. Сербин В. И. Марковская модель управления процессом обучения// XII Всеросс. совещ. по пробл. управления ВСПУ-2014 (16-19 июня 2014 г., Москва). - М.: Ин-т проблем управления им. В. А. Трапезникова РАН, 2014. - С. 9489-9497.

8. Соловов А. В. Электронное обучение: Проблематика, дидактика, технология. - Самара: Новая техника, 2006.

9. Харченко М. А. Корреляционный анализ. - Воронеж: Изд-во ВГУ, 2008.

10. Rasch G. Probabilistic Models for Some Intelligence and Attainment Tests. — Copenhagen, 1960.

11. Serbin V. Markov model of evaluation of learning results// Proc. 2018 Multidiscipl. Sympos. on Computer Science and ICT (Stavropol, Russia, October 15-19, 2018). — CEUR Workshop Proceedings, 2018. — Vol. 2254. - P. 114-122.

Сербин Владимир Иванович

Астраханский государственный университет

E-mail: V.I.Serbin@mail.ru 